Rev. Saúde públ., S. Paulo

9:551-3, 1975

\title{
PROGRAMA DE SAÚDE MENTAL
}

\author{
Cid Guimarães * \\ Eunice P. Castro Silva ** \\ Francisco Bernardini Trancredi* \\ José Maria Pacheco de Souza ** \\ Maria Helena Silveira ** \\ Ruy Laurenti** \\ Sabina Lea Gotlieb**
}

RSPU-B/293

Guimarães, C. et al. - Programa de saúde mental. Rev. Saúde públ., S. Paulo, 9:551-3, 1975 .

RESUMo: apresentado o programa de Saude Mental decorrente de convênio estabelecido pela Secretaria da Saúde do Estado de São Paulo com a Universidade de São Paulo, através da Faculdade de Saúde Pública. Este convênio tem por finalidade a realização de estudos na área de Saúde Mental, tais como assistência psiquiátrica preventiva e pesquisas visando a medir transtornos mentais através de casos de óbito e na população da cidade e de cursos $e$ atividades curriculares e extra-curriculares.

UNItermos: Saúde Mental, programa. São Paulo, SP (Brasil).

A Secretaria de Estado da Saúde, interessada em iniciar em São Paulo programas relativos à Saúde Mental, estabeleceu convênio com a Universidade de São Paulo, através da Faculdade de Saúde Pública, com o objetivo de:

1. ${ }^{\circ)}$ coletar dados epidemiológicos e sócio-culturais visando a permitir um melhor conhecimento dos fatores relacionados com a Saúde Mental;

$\left.2 .^{\circ}\right)$ desenvolver técnicas e recursos humanos na área da assistência psiquiátrica preventiva, em particular ambulatorial e no seio da comunidade.
Para alcançar a meta proposta, a execução do trabalho prevê cinco projetos específicos:

1. assistência psiquiátrica preventiva;

2. pesquisas na área da Saúde Mental;

3. cursos e atividades extra-curriculares;

4. cursos e atividades curriculares;

5. assistência técnica na área da Saúde Mental.

A assistência psiquiátrica preventiva tem seu projeto desenvolvido nos três ni-

* Do Departamento de Prática de Saúde Pública da Faculdade de Saúde Pública da USP - Av. Dr. Arnaldo, 715 - Săo Paulo, SP - Brasil.

* Do Departamento de Epidemiologia da Faculdade de Saúde Pública da USP - Av. Dr. Arnaldo, 715 - São Paulo, SP - Brasil. 
GUIMARAES, C. et al. - Programa de saúde mental. Rev. Saúde puibl., S. Paulo, 9:551-3, 1975.

veis de prevenção psiquiátrica, dando ênfase ao nivel primário, ao âmbito ambulatorial e na comunidade. Tem por objetivo instituir Serviço de Saúde Mental em Centro de Saúde polivalente, sendo escolhido o Centro de Saúde "Geraldo de Paula Souza" da Faculdade de Saúde Pública. Suas atividades, a cargo de equipe composta de médico psiquiatra, assistente social e psicólogo, englobarão consultas ambulatoriais, formação de grupos de orientação, através das populações de maior risco, consultas domiciliares e o estabelecimento de contatos com escolas e outras instituições.

A categoria programática representada por "pesquisas na área de Saúde Mental" "stá sendo desenvolvida através de dois projetos: "estudo dos transtornos mentais como causa básica ou associada na mortalidade em adultos de 15 a 74 anos de idade" e "estudo dos transtornos menlais e suas características na população do distrito de São Paulo".

() primeiro oljetiva fundamentalmente:

l.") determinar as reais causas Jásicas e associadas de morte em adultos dessa faixa etária;

2.") verificar a importância dos translornos mentais como causa básica e associada de morte;

3.") verificar a associação existente enltre doenças mentais e outras doenças: ;

1.") estudar algumas características de mortalidade segundo as causas e certas variáveis como sexo, idade, profissão, assistência médica, origem do falecido, entre outras.

Está sendo estudada uma amostra sistemática probabilística das mortes de residentes no distrito de São Paulo, com idades compreendidas entre 15 e 74 anos e ocorridas entre $1 .^{\circ}$ de outubro de 1974 e 30 de setembro de 1975. O estudo é baseado na análise detalhada de cada caso, feito através de entrevistas domiciliares e junto aos médicos, hospitais, etc., que tenham prestado assistência às pessoas falecidas.

A segunda pesquisa visa, em sintese:

1.") estudo da prevalência dos transtornos mentais na população;

2.) estudo da associação dos transtornos mentais a alguns fatores, como sexo, idade e condições sócio-econômico-cultural;

$3^{\circ}$ ) verificação dos meios procurados para tratamento dos transtornos mentais;

4. ${ }^{\prime \prime}$ verificação das condições de acesso à assistência psiquiátrica disponível;

5.") estudo da incidência dos transtornos mentais na população a partir de ohservação prospectiva.

() método de estudo consiste em investigar, em amostra probabilística de domicílios do distrilo de São Paulo, dados que: permitam atingir os objetivos propostos. Cada domicílio será considerado uma unidade amostral, sendo a amostra de aproximadamente 3.000 famílias.

Cursos e atividades extra-curriculares referem-se a treinamento de pessoal médico e de outros técnicos do setor saúde em práticas preventivas em Saúde Mental. Para tanto, seminários estão sendo programados, para realização tanto na Capital como no interior do Estado.

Cursos e atividades curriculares correspondem ao desenvolvimento da Disciplina de Higiene Mental, já oferecida pela Faculdade de Saúde Pública, que visa dar conhecimentos complementares sobre os problemas mais comuns de Saúde Mental. 
GUIMARAES, C. et al. - Programa de saúde mental. Rev. Saúde públ., S. Paulo, 9:551-3, 1975.

RSPU-B/293

Guimarães, C. et al. - [Programme in mental health]. Rev. Saúde públ., $S$. Paulo, 9:551-3, 1975.

SUMMARY: The School of Public Health is conducting a project, sponsored by the Health Department of the State of S. Paulo Government, aiming at: studying the integration of a team of mental health workers in a polivalent community health center; carrying out two large surveys on the incidence and prevalence of mental disorders and some of their characteristics; promoting courses and seminars on Mental Health for the personnel of Community Health Centers.

UNiterms: Mental Health, project. S. Paulo, SP (Brazil).

Recebido para publicaçāo em 08/09/1975

Aprovado para publicação em 22/09/1975 